\title{
Infrared and Visible Image Fusion Based on Nonparametric Segmentation
}

\author{
Harbinder Singh, P.N. Hrisheekesha, Gabriel Cristobal
}

\begin{abstract}
Image fusion is a process of combining an image sequence of the same scene into a single image for better human perception \& targeting. The thermal energy reflected from outstanding objects under poor lighting conditions and visible information that yield spatial details needs to be fused for improving the performance of surveillance systems. In this paper, we present a fusion technique that is helpful in surveillance systems for detecting targets when the background and the targets are of the same color. A nonparametric segmentation based weight map computation technique is proposed to extract target details from infrared (IR) imagery. The optimal threshold based on local features is selected automatically for target detection. With this, the extracted salient information of targets is blended to visible image without introducing distortions. The main advantage of the new technique is that it is based on a single-scale binary map (SSBM) fusion approach. The binary weight maps are computed for the fusion of separable IR target with visible imagery. An extension to IR and visible color image fusion is also suggested for target localization. Several simulation results are demonstrated for different data sets to support the validity of the proposed technique.
\end{abstract}

Index Terms: Infrared, visible image, night vision, image fusion, segmentation, histogram.

\section{INTRODUCTION}

The problem at hand aims to initiate research activities in night vision technology. It is imperative and critical for the army to have precise information about reaction time and accuracy in target detection. The proposed approach will be capable of fusing information from different electromagnetic bands which will result in an image lies within the human visual system and thus reducing reaction time in recognizing the enemy. Since resulting fused image will have information from more than one source it will have unnatural scene information. Though the fusion algorithm will minimize these effects but their complete removal is impossible. Though one may gain an intuitive feeling for image improvement simply by viewing fused night vision images before and after fusion but such intuitions are not quantifiable or precise. To minimize the effects of this unnatural information, we will test fused images for better perception.

With the widespread application in the defense and surveillance field, the fusion of IR imagery and image captured by $\mathrm{CCD}$ sensor become vital parts of the image

\section{Revised Manuscript Received on July 09, 2019.}

Harbinder Singh, Electronics and Communication Engineering, Chandigarh Engineering College, Landran, Mohali, India.

P.N. Hrisheekesha, Electronics and Communication Engineering, Chandigarh Engineering College, Landran, Mohali, India.

Gabriel Cristobal, Instituto de Optica, Spanish Council for Scientific Research (CSIC), Madrid, Spain. fusion technology which has developed speedily and received worldwide consideration of current research. Although infrared imaging sensors have the capability of identifying the thermal energy reflected from objects under poor lighting conditions. But it is less responsive to the changes of the scene light intensity variations and produces a low-quality image with fewer background details required for human perception. On the other hand, visible charge coupled devices (CCD) sensor is totally dependent on light intensity variations present in the target scene. Therefore, a better image can be generated with higher clarity through the fusion of IR imagery and visible imagery.

Motivated by multi-modal image data sets obtained from an infrared sensor and visible light sensor: the thermal energy reflected from objects under poor lighting conditions and visible information that yield spatial details can be fused to improve the performance of surveillance systems. This fusion process preserves background information from visual light image and target details from an infrared image that improves acquiring, targeting and identification performance. Moreover, this will be helpful in military surveillance systems for detecting targets when the background and the targets are o the same color.

The standard camera has CCD sensors which are capable of capturing visual range information. While for capturing information out of visual range we require specialized cameras. For example, an IR sensor camera will be able to capture information pertaining to heat patterns from living beings. Fusion is a technique which links information from two or more type of sensors or varying exposure level images and generates a single image containing overall information. While linking or fusing information it makes sure that the overall information quality of the picture is increased.

\section{RELATED WORK}

\section{A. Thermal Imaging}

Inspired by the fact that thermal imaging camera captures wavelengths up to $14,000 \mathrm{~nm}$ instead of a visible light wavelength of 400-700 nm. In 1800, Sir William Herschel has proposed the utilization of infrared for detecting radiations beyond red light. In recent years, various IR based devices have been developed for the civil section [1]. The principal motivation for thermal imaging is to detect radiations from objects under the influence of poor light conditions. The first infrared camera was invented in 1929 for anti-aircraft defense in Britain [2]. The thermal imaging has been widely accepted as one of the most effective techniques for

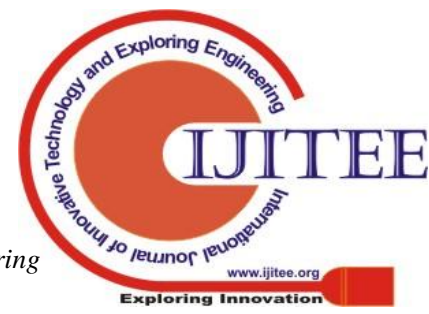


security systems because IR imagery is nearly invariant to variations in ambient illumination [3], which is useful for identifying and targeting the objects under the influence of different illuminations including total darkness [3].

\section{B. Image Fusion}

In recently proposed work [4], multi-exposure image fusion techniques have been developed to handle the High Dynamic Range (HDR) scene. Exposure fusion approach has been found as a solution to combine details of an object under the influence of different lighting conditions. In particular, it overcomes the limitation of conventional digital camera that is not able to capture the interior and exterior of a building simultaneously. Therefore, the fusion of images captured by a single sensor at different exposure settings would be able to simultaneously record indoor as well as outdoor activities. The fusion night vision system [5] has been proposed that combine image intensification with thermal sensing. The image intensification information and the infrared information are fused together to produce a fused image that provides the benefits over just image intensification or just thermal sensing. More recently, a fusion of different sensor data sets was shown to provide better surveillance system than a system using a single imaging sensor. In this paper, we focused on solving the problem of preserving and enhancing details by fusing information captured by the visible camera and thermal imaging camera. The main steps involved in the framework of the proposed approach are described in Algorithm 1, which is described in the forthcoming section. In order to evaluate the performance of the present approach, a variety of image data sets are considered in experimental results.

\section{NONPARAMETRIC SEGMENTATION FOR TARGET DETECTION AND WEIGHT MAP CPMPUTATION}

In IR imagery, the foreground target region has unique characteristics, which is helpful to identify and analyze the target from the background. As can be observed in the experimental results illustrated in Figs. 1 (a-e), the global histogram consideration may become less effective for IR target separation in complex background. This is because IR images are based on heat induction. The higher intensity levels represent a higher temperature for unique hot targets. Therefore, a histogram of separable IR imagery yields a unimodal peak as illustrated in Fig 1 (d), so the target separation to be achieved by adaptive threshold based segmentation technique (see Fig. 1 (e)). The target localization by combining visible and infrared sensors is directly affected by the image segmentation. Various image segmentation methods [6] have been developed in recent years. In the present approach, we assume that foreground target objects have grouped into one dominant mode.

In this section, the binary weight maps for IR target separation are computed by an improved global thresholding method at first. Then the background details from visible light imagery and target region from IR imagery are fused for situational awareness.

\section{A. Optimum Threshold Selection for Target Detection}

The nonparametric segmentation is a general class of global thresholding methods, which select the optimum threshold from gray-level histogram [7]. This type of segmentation method can be useful for object separation, where the histogram of the image is bimodal [8]. Another class of segmentation based on region growing [region growing] having identical features are also suitable for this type of applications. We extend the global thresholding technique [7] here, in which the threshold is determined by considering the unique characteristics of the target region. The main idea of the proposed simplex algorithm is to identify target regions in an IR image that have higher intensity levels with unique characteristics.

Let $f_{\text {ir }}(x, y)$ be the IR image, which is inspected by IR sensor and $f_{v i}(x, y)$ is the visible light image inspected by CCD sensor for $(x, y) \in \Omega$, and are assumed to be co-registered. The pixels of $f_{i r}(x, y)$ be represented in $L$ gray levels in the range [1, $2,3, \ldots \ldots, L]$.

To consider the target region characteristics, first, the $f_{i r}$ $(x, y)$ image is divided into a minute region of constant intensity levels belong to global maxima. In the present approach $\operatorname{fir}(x, y)$ is divided into $N \times N$ small region based on global maxima corresponds to the target region. We set the maximum window size as $6 \times 6$ (i.e. $N=6$ ). In order to improve the performance of the present approach, small IR target detection methods [9] can be used to locate IR targets against different complex backgrounds. Suppose there are $K$ different objects $R_{l}, R_{2}, \ldots \ldots, R_{k}, \ldots \ldots R_{K}$, and $R_{k}$ is the region contains global maxima. Let the range of pixels of a region of interest $R_{k}$ be represented in $\ell$ gray levels in the range $[g, \ldots ., \ell]$.

After locating the target region $R_{k}$, the histogram of the same region is denoted by:

$p_{q}=\frac{n_{q}}{n} \quad q=g, \ldots ., \ell$

where $n$ is the total number of pixels in the target region with gray level range $[g, \ldots . ., \ell], n_{q}$ is the number of pixels having

gray levels $q$, and $\ell$ is the total number of possible gray levels. Now, suppose a threshold $\kappa$ is chosen to dichotomized the gray levels range $[g, \ldots . ., \ell]$ into two classes $C_{0}$ and $C_{l}$ in the gray level range $[g, \ldots . ., \kappa]$ and $[\kappa+1, \ldots ., \ell]$, respectively. Otsu's method [7] chooses the optimal threshold $\kappa$ that maximizes the between-class variance $\sigma_{B}^{2}(\kappa)$, defined as

$\sigma_{B}^{2}(\kappa)=P_{1}(\kappa)\left[m_{1}(\kappa)-m_{G}\right]^{2}+P_{2}(\kappa)\left[m_{2}(\kappa)-m_{G}\right]^{2}$

where $P_{1}(\kappa)$, and $P_{2}(\kappa)$ are the probabilities of set $C_{l}$, and

$C_{2}$, respectively, and are given by

$$
\begin{aligned}
& P_{1}(\kappa)=\sum_{i=g}^{\kappa} p_{i} \\
& P_{2}(\kappa)=\sum_{i=\kappa+1}^{\ell} p_{i}=1-P_{1}(\kappa)
\end{aligned}
$$

Similarly, the terms $m_{1}(\kappa)$, and $m_{2}(\kappa)$ are the mean intensities of the pixels in set $C_{1}$, and $C_{2}$, respectively, and $m_{G}$ is the global mean of selected target region $R_{k}$ belongs to global maxima of 
the entire IR image, and are define as follows

$$
\begin{aligned}
& m_{1}(\kappa)=\sum_{i=g}^{\kappa} i p_{i} \\
& m_{2}(\kappa)=\sum_{i=\kappa+1}^{\ell} i p_{i} \\
& m_{G}=\sum_{i=g}^{\ell} i p_{i}
\end{aligned}
$$

By expanding the expression for $\sigma_{B}^{2}(\kappa)$, and using

(3), between-class variance [8] in (2) can be defined as

$$
\sigma_{B}^{2}(\kappa)=\frac{\left[m_{G} P_{1}(\kappa)-m(\kappa)\right]^{2}}{P_{1}(\kappa)\left[1-P_{1}(\kappa)\right]}
$$

The expression in (4) is computationally efficient than expression in (2) because only two parameters, $m$ and $P_{l}$ have $\kappa$ calculated in (5) is used as a threshold $T$ for binary weight map commutation in the forthcoming section. The basic steps involved in the proposed approach are listed in Algorithm 1.

Algorithm 1. Basic steps used in proposed algorithm Input: IR $f_{I R}(x, y)$ and visible $f_{v i}(x, y)$ images Output: Fused image.

1. Construct the de-noised IR image of $f_{I R}(x, y)$ from (6).

2. Construct the $N \times N$ region $R_{k}$ corresponds to global maxima.

3. Construct histogram of target region $R_{k}$ from (1).

4. Compute the value of threshold $T$ from (5).

5. Construct the binary mask $W_{i r}(x, y)$ for IR image from (7).

6. Construct the binary mask $W_{v i}(x, y)$ for visible image from (8).

7. Construct fused image $R_{k}$ from (9).

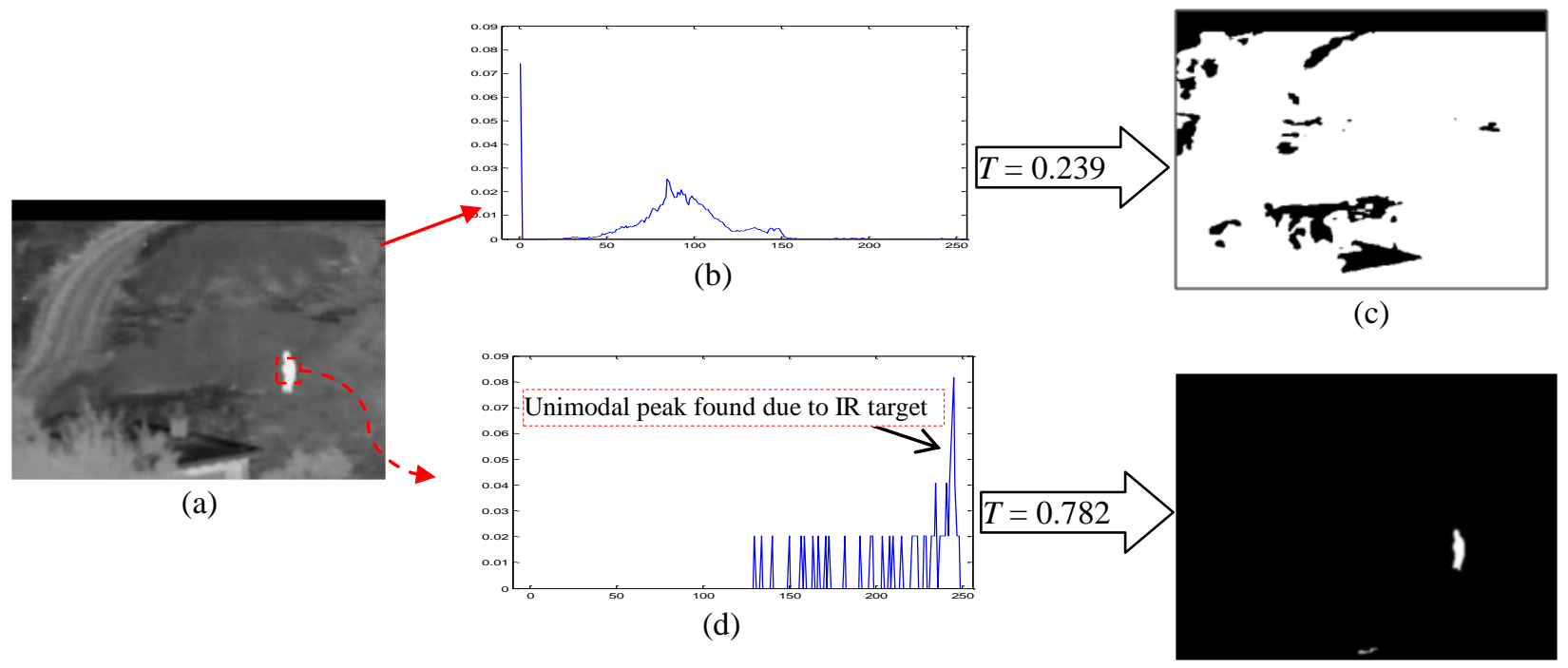

(e)

Fig. 1. Probability distribution function (PDF) and binary weight map computation. (a) input IR image "UN Camp", (b) PDF of IR image, (c) binary weight map computed based on threshold $(T)=0.239$, (d) PDF of IR target region corresponds to global maxima, and (e) weight map computed based on threshold $(T)=0.782$.

to be computed for all values of $\kappa$ and $m_{G}$ is computed for target region $R_{k}$ only once.

The thought of maximizing the between-class variance is that the larger this variance is, the more likely it is that threshold will segment the target region properly. This optimality measure depends on the histogram computed for the target region based on global maxima. In addition, because $\kappa$ is an integer in the range $[g, \ldots . ., \ell]$. The optimum value of $\kappa$ is selected by finding the maximum of $\sigma_{B}^{2}(\kappa)$ for gray values related to the target region. If the maximum is not unique, the threshold used is the average of all the optimum $\kappa^{\prime} s$ found. The measure of separability $\eta(\kappa)$ of image intensities into two classes (e.g. target region and background) can be defined as

$\eta(\kappa)=\frac{\sigma_{B}^{2}(\kappa)}{\sigma_{G}^{2}}$

It is noticed that the value of $\eta(\kappa)$ lies between 0 and 1 . It yields minimum value if the pixels of the target region are inseparable from the background.

Intuitively, it is expected that as more emphasis is placed on the threshold determination by considering the unique characteristics of the target region. The optimum value of
Fig. 2 (a) shows that the threshold value computed with simple Otsu method is significantly lower than the threshold value $(T)$ computed with the proposed approach. Higher the threshold values better the segmentation of IR targets. In this experiment, an adaptive threshold $(T)$ was computed for five data sets. Proposed segmentation approach computes higher threshold values for all five IR data sets automatically, in which the target has unique intensity variations. The effectiveness measure computed for Otsu method [7] and the proposed approach for the same IR data sets are shown in Fig. 2 (b). It can be observed from Fig. 2 (b) that proposed approach yields a higher value of effectiveness measure compared to the base method.

\section{B. Effect of Noise}

The IR images are far noisier than the visible light images. In the absence of noise, the global maxima are determined for separable target region $R_{k}$ perfectly. In a noisy IR image, the noise and pixel contain information are not well separated. The noise present in the IR image yield wrong selection of a threshold for target segmentation. One solution to this problem is to attempt to apply de-noising. In the

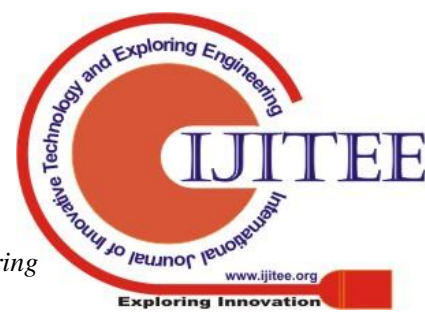



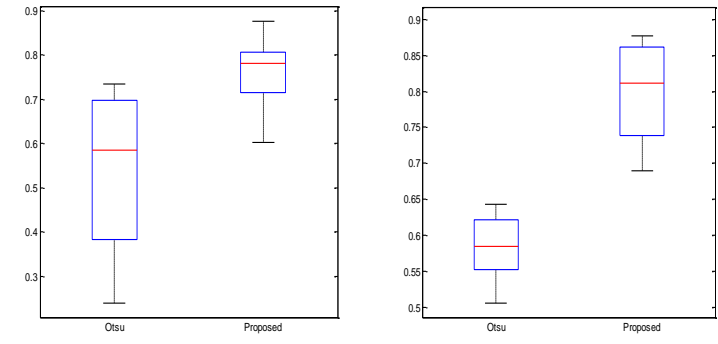

Fig. 2. Box plot of threshold value $(T)$ and effectiveness measure for five data sets ("UN Camp", "Dune", "Bunker", "Bench", "Kaptein") (a) variations of threshold $(T)$ computed by Otsu [7] and proposed method, and (b) effectiveness measure computed for Otsu [7] and proposed method

present approach, IR image is filtered by Gaussian smoothing prior to the selection of the target region and maximizing between-class variance $\sigma_{B}^{2}(\kappa)$ in (4). The Gaussian function with normal distribution is defined by

$$
G(x, y)=\frac{1}{2 \pi \sigma^{2}} e^{-\frac{x^{2}+y^{2}}{2 \sigma^{2}}}
$$

where $x$ represents the distance from the center of the local window corresponds to a pixel in interest in the horizontal direction, $y$ represents the distance from the center of the same local window in the vertical direction, and $\sigma$ is the standard deviation of the Gaussian distribution. It has been found that 3 by 3 convolution kernel approximating a Gaussian function with $\sigma$ of 0.5 is more appropriate for threshold detection.

In Fig. 1 (b), we can observe that thresholding is likely to fail if noise is present and the computed threshold value does not fit into target present in the input IR image.

\section{Binary Weight Map Computation}

In the present approach, the optimum threshold $T$ value computed in (5) is used for binary mask computation. The mask for IR is defined as follows

$$
W_{i r}(x, y)= \begin{cases}1 & \text { if } f_{i r}(x, y)>T \\ 0 & \text { if } f_{i r}(x, y) \leq T\end{cases}
$$

and binary mask for a visible image is defined by

$W_{v i}(x, y)=1-W_{i r}$

Further to avoid artifacts near IR target regions during the fusion process, weight map computed in (7) are optimized using [10]. The binary weight maps computed for four image data sets are illustrated in Fig. 2.

\section{SINGLE-SCALE IR AND VISIBLE IMAGE FUSION}

Once the resulting binary maps $W_{i r}(x, y)$ and $W_{v i}(x, y)$ are obtained, the pixelwise single-scale composition of $f_{i r}(x, y)$ and $f_{v i}(x, y)$ is calculated as follows

$$
f_{F}(x, y)=W_{i r}(x, y) \times f_{i r}(x, y)+W_{v i}(x, y) \times f_{v i}(x, y)
$$

\section{EXPERIMENTAL STUDY AND ANALYSIS}

Four experimental data sets of IR and grayscale visible imagery pairs are chosen for discussion, which is shown in Fig. 3. Firstly, the experimental results of grayscale data sets with comparison are introduced; then the discussion of fusion results for two IR and visible color imagery pairs will be
Based on Nonparametric Segmentation

discussed. The first series of images are of "Bench" which comprises of a bench, river, fencing, and path (see Fig. 3 (a), and Fig. 3 (b)). In the fused image (see Fig. 3 (c)) the target is perfectly embedded into the visible image for situational awareness. The second series of images are of "Kaptein" which comprises surveillance of building area. It is very difficult to distinguish a person moving along the trees in the visible image (Fig. 3 (f)). On the other hand, the position of a target under surveillance is visible in the IR image (Fig. 3 (g)). We can observe that the proposed resultant fused image yields background and target details for guarding purpose.

In the third data set 'Bunker' image data set corresponds to surveillance and detection of bunker hidden in the trees. In the visible image, the bunker is not clearly detectable and in IR the background details are missing (see Fig. 3 (k) and Fig. 3 (1)). After fusion, the position of the bunker can be determined relative to background details.

The fourth data used in the experiment is the image series of 'UN Camp'. In the visible image, the fencing used to enclose defense area, and trees are visible (Fig. 3 (p)). It is always desirable to monitor a fenced area and locate the position of IR target (Fig. 3 (q)) moving along the fence for any intrusion. The proposed fusion approach transfers the details of IR target and background details to the fused image for situational awareness.

In order to demonstrate the effectiveness, proposed fusion results are visually compared to three recently proposed approaches, namely, [11], [12], and [13]. The results of [11] for five data sets are shown in Fig. 4 (b, f, j, n, and r). The results of [12] for similar data sets are shown in Fig. 4 (c, g, k, $\mathrm{o}$, and s), and the results of [13] are shown in Fig. 4 (d, h, l, p, and $\mathrm{t}$ ). The results of the proposed approach for similar image series are illustrated in Fig. 4 (a, e, i, m, and q). After visual inspection of different aforementioned methods, we can observe that proposed fusion results preserve best target details without affecting the background's spatial information.

Moreover, the proposed fusion approach is 
implemented for grayscale IR and color visible image data set fusion. As we can notice from $(8), W_{v i}(x, y)$ is simply the inversion of $W_{i r}(x, y)$. For color image fusion, identical weight maps (i.e. $\left.W_{v i}(x, y)\right)$ are utilized for Red, Green and Blue channels. For illustration purpose, the fusion results for color visible image data sets are shown in Fig. 5. The detection of human activities in fused image from noisy and incomplete data set (i.e. smoky conditions, please see Fig. $5(\mathrm{a}, \mathrm{b})$ ) is shown in Fig. 5 (c). The proposed fused image from color visible image and grayscale infrared image captured under poor lighting conditions is shown in Fig. 5 (d). Therefore, the proposed approach produces better fused image with higher clarity through the fusion of grayscale IR and color visible imaging technology.

TABLE I. OBJECTIVE ANALYSIS BASED ON FRMSE AND CC COMPUTATION

\begin{tabular}{|c|c|c|c|c|c|}
\hline $\begin{array}{c}\text { Quality } \\
\text { Metric }\end{array}$ & $\begin{array}{c}\text { Image } \\
\text { data sets }\end{array}$ & Our & $\begin{array}{c}\text { Context } \\
{[\mathbf{9}]}\end{array}$ & KL[10] & $\begin{array}{c}\text { Two } \\
{[\mathbf{1 3}]}\end{array}$ \\
\hline \multirow{4}{*}{ FRMSE } & UN camp & $\mathbf{9 . 4 5 0}$ & 83.212 & 22.469 & 25.107 \\
\cline { 2 - 6 } & Bunker & $\mathbf{2 2 . 1 5 3}$ & 45.718 & 28.633 & 28.595 \\
\cline { 2 - 6 } & Dune & $\mathbf{3 . 5 8 6}$ & 38.302 & 14.077 & 13.563 \\
\cline { 2 - 6 } & Bench & $\mathbf{2 3 . 3 9 7}$ & 68.834 & 41.349 & 39.647 \\
\cline { 2 - 6 } & Kaptein & $\mathbf{1 6 . 7 6 5}$ & 61.551 & 39.198 & 39.267 \\
\hline \multirow{4}{*}{ CC } & UN camp & $\mathbf{0 . 9 7 5}$ & 0.835 & 0.861 & 0.816 \\
\cline { 2 - 6 } & Bunker & $\mathbf{0 . 8 9 2}$ & 0.869 & 0.834 & 0.802 \\
\cline { 2 - 6 } & Dune & $\mathbf{0 . 9 8 5}$ & 0.816 & 0.748 & 0.764 \\
\cline { 2 - 6 } & Bench & $\mathbf{0 . 9 3 7}$ & 0.843 & 0.886 & 0.845 \\
\cline { 2 - 6 } & Kaptein & $\mathbf{0 . 9 6 0}$ & 0.835 & 0.780 & 0.753 \\
\hline
\end{tabular}

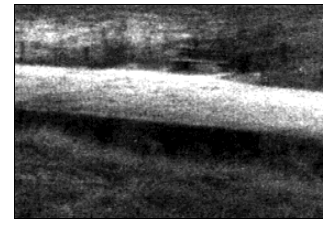

(a)

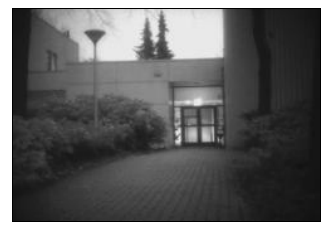

(f)

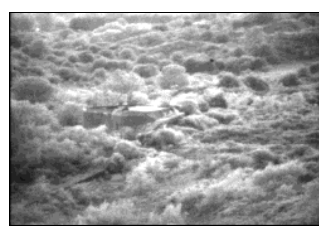

(k)

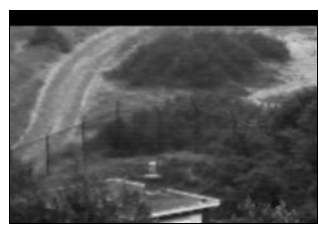

(p)

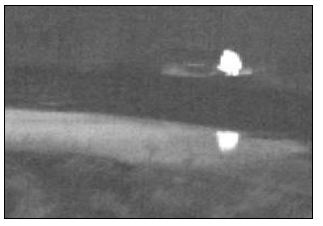

(b)

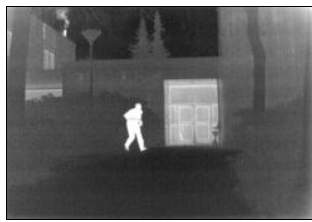

(g)

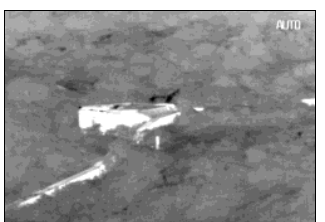

(1)

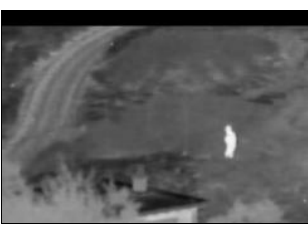

(q)

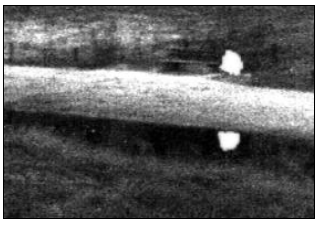

(c)

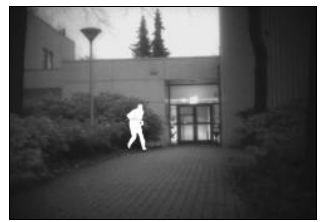

(h)

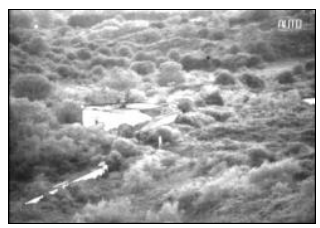

(m)

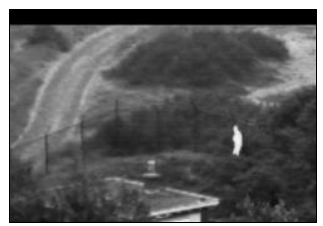

(r)

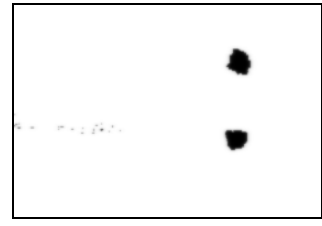

(d)

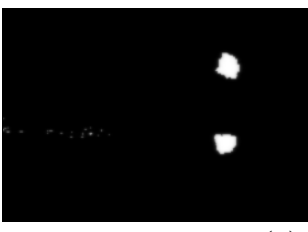

(e)

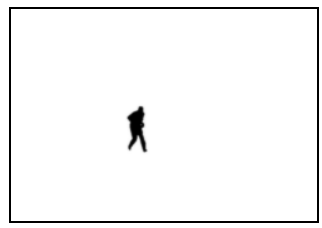

(i)

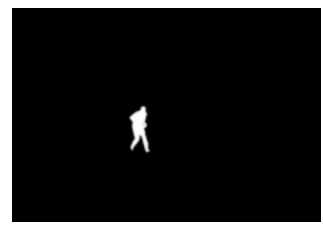

(j)

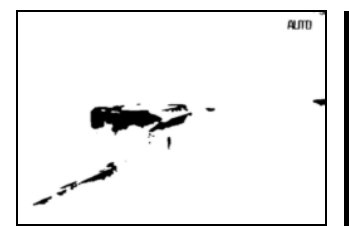

(n)

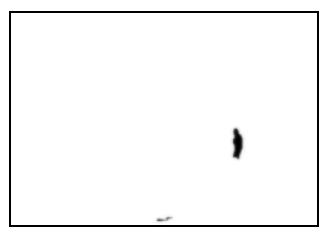

(s)

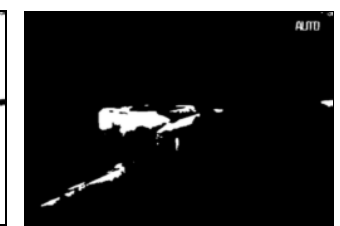

(o)

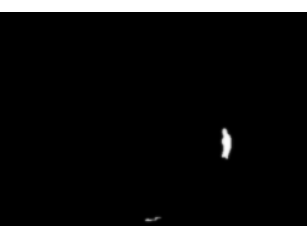

(t)

Fig. 3. Visual inspection of proposed fusion results. "Bench": (a) visible image, (b) IR image, (c) fused image, (d) weight map of visible image, and (e) weight map of IR image. "Kaptein": (a) visible image, (b) IR image, (c) fused image, (d) weight map of visible image, and (e) weight map of IR image. "Bunker": (a) visible image, (b) IR image, (c) fused image, (d) weight map of visible image, and (e) weight map of IR image. "UN Camp": (a) visible image, (b) IR image, (c) fused image, (d) weight map of visible image, and (e) weight map of IR image.

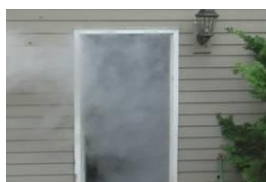

(a)

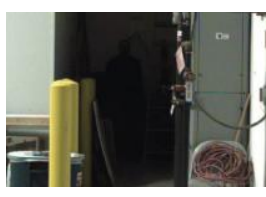

(c)

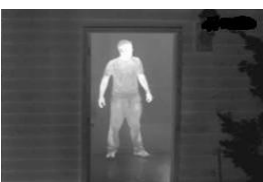

(b)

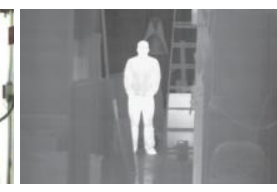

(d)

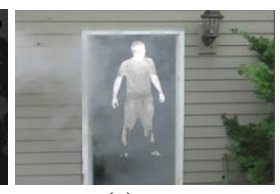

(c)

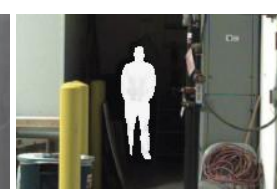

(e)

Fig. 5. Fusion results for IR and visible color imagery data sets. "Smoke": (a) visible image, (b) IR image, and (c) fused image. "Man": (c) visible image, (d) IR image, and (e) fused image.

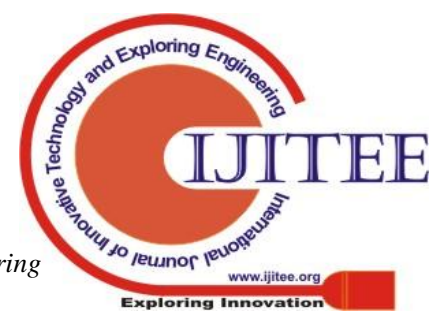




\section{A. Performance Analysis of Fusion Results}

In order to evaluate the performance of fusion approach, the quantitative analysis is given in Table I. In present approach the salient IR target is embedded in the visible image and in most of the cases the size of the target is small relative to background spatial information. Therefore, a visible image is to be considered as an ideal reference for objective analysis. However, Fusion Root-mean Square (FRMSE) and correlation coefficient (CC) metrics [14, 15] are used to evaluate the fusion performance. The superior values of the proposed approach for all five input data sets in Table I prove that the proposed fusion results have a strong correlation to input visible image. Therefore, the target and background are perfectly preserved in the fused image.

\section{CONCLUSION}

An efficient single-scale binary map (SSBM) fusion approach is proposed to fuse details from visible and infrared images. The proposed approach achieves good results for situational awareness when the background and targets are of the same color under poor lighting conditions. Moreover, for better perception and detection of human activities through color image fusion from noisy and incomplete data set (i.e. smoky conditions) is also discussed. A new binary weight map computation method is proposed using nonparametric segmentation. The objective analysis of proposed fusion results proves that the target and background are perfectly preserved in the fused image.

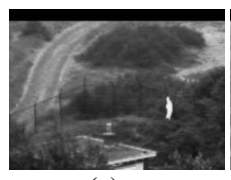

(a)

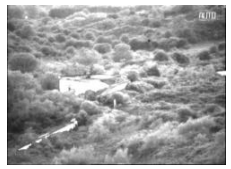

(e)

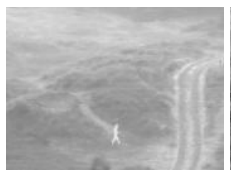

(i)

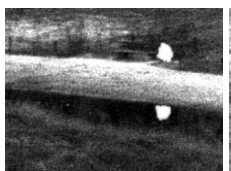

(m)

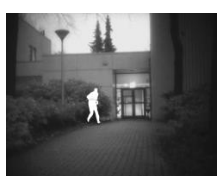

(q)

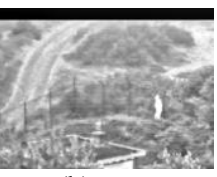

(b)

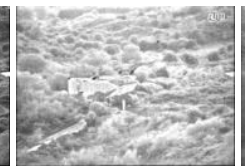

(f)

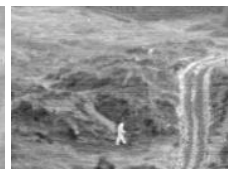

(j)

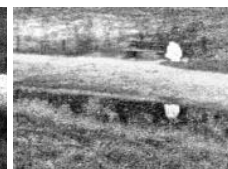

(n)

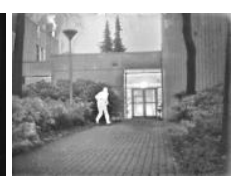

(r)

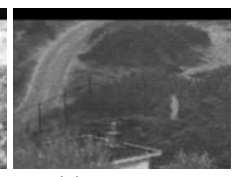

(c)

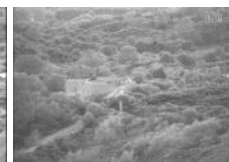

(g)

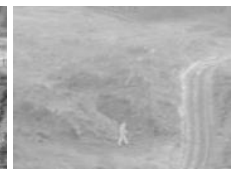

(k)

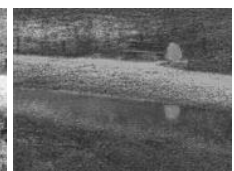

(o)

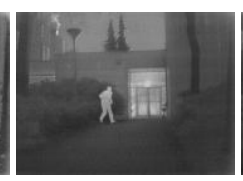

(s)

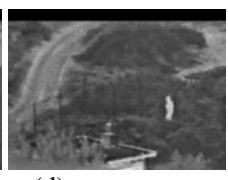

(d)

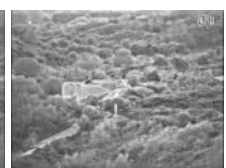

(h)

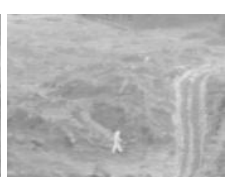

(1)

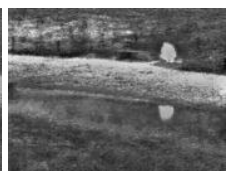

(p)

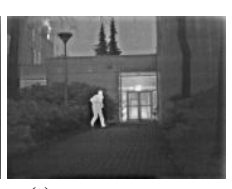

(t)
Fig. 4. Comparison of experimental fusion results. "UN Camp": (a) proposed, (b) context [11], (c) KL [12], and (d) Two scale [13]. "Bunker": (e) proposed, (f) context [11], (g) KL [12], and (h) Two scale [13]. "Dune": (i) proposed, (j) context [11], (k) KL [12], and (l) Two scale [13]. "Bench": (m) proposed, (n) context [11], (o) KL [12], (p) Two scale [13]. "Kaptein": (q) proposed, (r) context [11], (s) KL [12], and (t) Two scale [13].

\section{REFERENCES}

1. A. Toet, J.K. IJspeert, A.M. Waxman, and M. Aguilar, "Fusion of visible and thermal imagery improves situational awareness," Displays, 18, pp. 85-95, 1998.

2. Kalman Tihanyi, Monash University, Archived from the original on 24 October 2003. Retrieved 15 March 2013.

3. Y. Niu, S. Xu, L. Wu, and W. Hu, "Airborne infrared and visible image fusion for target perception based on target region segmentation and discrete wavelet transform," Mathematical Problems in Engineering, vol. 2012, Article ID 275138, 10 pages, 2012.

4. H. Singh, V. Kumar, and S. Bhooshan, "Anisotropic diffusion for details enhancement in multiexposure image fusion," ISRN Signal Processing, vol. 2013, Article ID 928971, 18 pages, 2013.

5. S. Li, X. Kang, J. Hu, and B. Yang, "Image matting for fusion of multi-focus images in dynamic scenes," Information Fusion, vol. 14, no. 2, pp. 147-162, 2013.

6. P. K. Saho, S. Soltani, and A. K. C. Wong, "A Survey of Thresholding Techniques," Computer Vision, Graphics, and Image Processing, vol 41, pp. 233-260, 1988

7. N. Otsu, "A Threshold Selection Method from Gray-Level Histograms," in IEEE Transactions on Systems, Man, and Cybernetics, vol. 9, no. 1, pp. 62-66, Jan. 1979.

8. R. C. Gonzalez, and R.E. Woods, Digital Image Processing, $3^{\text {rd }}$ edition, Prentice Hall, 2008.

9. C. Yang, J. Ma, S. Qi, J. Tian, S. Zheng, and X. Tian, "Directional support value of Gaussian transformation for infrared small target detection," Applied Optics, vol. 54, no. 9, pp. 2255-2265, 2015.

10. H. Singh, V. Kumar, and S. Bhooshan, "Weighted Least Squares Based Detail Enhanced Exposure Fusion,” ISRN Signal Processing, vol. 2014 Article ID 498762, 18 pages, 2014. H. Singh, V. Kumar, and S. Bhooshan, "Weighted Least Squares Based Detail Enhanced Exposure Fusion," ISRN Signal Processing, vol. 2014, Article ID 498762, 18 pages, 2014.

11. Zhiqiang Zhou, Mingjie Dong, Xiaozhu Xie, Zhifeng Gao "Fusion of infrared and visible images for night-vision context enhancement," Appl Opt., vol. 55, no. 23, pp. 6480-6490, 2016.

12. D. P. Bavirisetti and R. Dhuli, "Fusion of Infrared and Visible Sensor Images Based on Anisotropic Diffusion and Karhunen-Loeve Transform," in IEEE Sensors Journal, vol. 16, no. 1, pp. 203-209, Jan.1, 2016

13. Durga Prasad Bavirisetti and Ravindra Dhuli "Two-scale image fusion of visible and infrared images using saliency detection," Infrared Physics \& Technology, ISSN: 1350-4495, vol. 76, pp. 52-64, 2016.

14. H Singh, H. Fatima, S. Sharma and D. Arora, "A Novel Approach for IR Target Localization Based on IR and Visible Image Fusion," IEEE 4th International Conference on Signal Processing, Computing and Control (ISPCC 2017), pp. 235 - 240, September 21-23, 2017.

15. Zi-Jun Feng, Xiao-Ling Zhang, Li-Yong Yuan, and Jia-Nan Wang, "Infrared Target Detection and Location for Visual Surveillance Using Fusion Scheme of Visible and Infrared Images," Mathematical Problems in Engineering, vol. 2013, Article ID 720979, 7 pages, 2013.

\section{AUTHORS PROFILE}

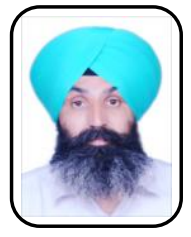

Harbinder Singh has done Ph.D. degree in Electronics and Communication Engineering at the Jaypee University of Information Technology, Waknaghat, India, received the M. Tech degree in Electronics and Communication Engineering from Punjab Technical University, Punjab, the $\mathrm{B}$. Tech degree in Electronics and Communication Engineering from Himachal Pradesh University, Shimla and 3-year diploma in Electronics and Communication Engineering from Punjab State Board of Technical Education. He is the author or co-author of 24 papers published in national, international conferences and journals. He has few patents and book chapters to his credit. His current research interests include diatom analysis based on multi focus image fusion, exposure fusion, edge-preserving filters, high dynamic range imaging and tone mapping. $\mathrm{He}$ is currently working as Associate Professor, ECE, Chandigarh Engineering College Landran, Mohali, India.

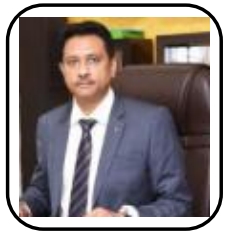

P.N. Hrisheekesha is a B. E, M. Tech, Ph. D from IIT Roorke. Currently he is working as campus director at 
CGC Landran. Has got more than 50 publications in reputed international and national journals and conferences. He has completed so many funded projects. He is the editor of few reputed journals. He has guided more than 50 PG and few Ph.Ds. He has few patents and books to his credit. He has established many incubation centers, Center of innovation, new labs too. His area of research and interest are renewable energy sources, power systems, distribution system automation, image processing, energy conservation, audit, printed electronics, AI, machine learning.

Gabriel Cristobal is a Research Scientist of the

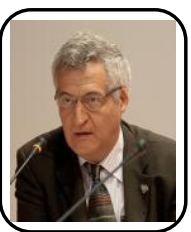
Institute of Optics (CSIC). During the period 1982-89 he was a Collaborating Professor and later Associated Professor at the Polytechnic University of Madrid. In 1989-91 he was Visiting Scholar at the International Computer Science Institute (USA) and in 1991 he was a researcher hired at the Electronics Research Lab (UC Berkeley). His research interest are the development of image analysis methods in microscopy, vision modelling and image quality assessment. He has been responsible for several national and European research and development projects. Among them is the ADIAC project (1998-2001), which was the first European pilot project in the area of diatom identification. Currently is the coordinator of the AQUALITAS project on automatic diatom identification (CTM2014-51907). He was a Faculty Member of the Madrid-MIT M+ Vision Consortium dedicated to medical imaging research. 